\title{
Calidad de forraje y almidón en 10 clones de batata Ipomoea batatas (L.) Lam.
}

\section{Forage and starch quality in $\mathbf{1 0}$ sweet potato clones Ipomoea batatas (L.) Lam.}

\author{
Magda Piedad Valdés-Restrepo ; Sanin Ortiz-Grisales ${ }^{2}$
}

${ }^{1}$ Ing. Agroindustrial; Ing. Agrónoma; M.Sc.; Ph.D. Universidad Nacional Abierta y a Distancia. Palmira - Valle del Cauca, Colombia; e-mail: magda.valdes@unad.edu.co; iDhttps://orcid.org/0000-0001-9594-0289

2 Zootecnista; M.Sc.; Ph.D. Universidad Nacional de Colombia. Palmira - Valle del Cauca, Colombia; e-mail: sortizg@unal.edu.co; iD https://orcid.org/0000-0002-7237-0815

Cómo citar: Valdés-Restrepo, M.P.; Ortiz-Grisales, S. 2021. Calidad de forraje y almidón en 10 clones de batata Ipomoea batatas (L.) Lam. Rev. U.D.C.A Act. \& Div. Cient. 24(2):e1580. http://doi.org/10.31910/rudca.v24.n2.2021.1580

Artículo de acceso abierto publicado por Revista U.D.C.A Actualidad \& Divulgación Científica, bajo una Licencia Creative Commons CC BY-NC 4.0

Publicación oficial de la Universidad de Ciencias Aplicadas y Ambientales U.D.C.A, Institución de Educación Superior Acreditada de Alta Calidad por el Ministerio de Educación Nacional.

Recibido: mayo 29 de $2020 \quad$ Aceptado: agosto 26 de 2021 Editado por: Helber Adrián Arévalo Maldonado

\section{RESUMEN}

La batata Ipomoea batatas (L.) Lam. es una manifestación de la biodiversidad silvestre y cultivada del Neotrópico, caracterizada por hojas anchas y tubérculos feculentos. La especie es objeto de investigación, por su amplio uso e importancia en regiones tropicales y subtropicales, por sus propiedades nutricionales y aporte energético, convirtiéndose en un recurso imprescindible para la seguridad alimentaria. El objetivo de esta investigación fue evaluar el rendimiento agronómico, en valor relativo del forraje (VRF) y rendimiento y calidad de almidón, en tubérculos de batata. Se evaluaron 10 clones seleccionados por alta materia seca en tubérculos, con un diseño de bloques completos al azar, con 3 repeticiones y 6 plantas por repetición. Se destacó el clon 440-078, con un VRF de 133,07 y por el alto rendimiento de tubérculos los clones 440-656 y 440-621, considerados como materiales promisorios para calidad del tubérculo; este último presentó el peso de tubérculo más alto en las tres categorías identificadas A, B y C. El clon Tainung-66, se posicionó con -1.53 de ISP, asociado con bajos valores de fibra (28,9 y 20,25 de FDN y FDA, respectivamente), que le confieren una digestibilidad burda del tubérculo del $72 \%$.
Además, se determinaron diferencias significativas $(p<0,05)$, en las propiedades de empastamiento del almidón, con superioridad del clon 440-016, por sus características retrogradantes, con $221 \mathrm{cP}$ y el clon Tainug-66, por su estabilidad teniendo en cuenta la viscosidad.

Palabras clave: Alimentación animal; Alimento funcional; Evaluación agronómica; Fitomejoramiento; Tubérculos.

\section{ABSTRACT}

The sweet potato Ipomoea babatas (L.) Lam., is a manifestation of the wild and cultivated Neotropical biodiversity, characterized by leaves with value as forage and starchy tubers, it is the object of research for its wide use and importance in tropical and subtropical regions, for its nutritional properties and contribution energy, becoming an essential resource for food security. The agronomic yield was evaluated in relative forage value (RFV), yield, and starch quality in sweet potato tubers. 10 clones selected for high dry matter in tubers were evaluated, with a randomized complete block design with 3 repetitions and 6 plants per repetition. Clone 440-078 stood out with an RFV of 133.07 and due to its high performance in tubers, 
Valdés-Restrepo, M.P.; Ortiz-Grisales, S.: evaluación de clones de batata

clones 440-656 and 440-621, considered as promising materials for tuber quality, the latter presented the highest tuber weight in the three identified categories A, B, and C. The Tainung-66 clone is positioned in first place with -1.53 ISP associated with low fiber values (28.9 and 20.25 of $\mathrm{NDF}$ and ADF respectively), which give it a gross tuber digestibility of $72 \%$. Significant differences $(\mathrm{p}<0.05)$ in starch plastering properties were determined, with superiority at clone 440-016 for its retrograde characteristics with $221 \mathrm{cP}$ and the Tainug- 66 clone for its stability taking into account the maximum viscosity $(\mathrm{Vm})$ and the viscosity of the cold paste (VPF) with $730 \mathrm{cP}$ and $738 \mathrm{cP}$ respectively.

Keywords: Animal feeding; Agronomic evaluation; Functional foods; Plant breeding; Tubers.

\section{INTRODUCCIÓN}

Batata Ipomoea batatas (L.) Lam. (Convolvulaceae), una manifestación de la biodiversidad silvestre y cultivada, una planta perenne dicotiledónea, que produce raíces tuberosas comestibles. Es la única especie de importancia económica como cultivo alimenticio del género Ipomoea, y uno de los cultivos de raíces de mayor importancia, a nivel mundial (Koussao et al. 2014), con una producción anual de 91.945.358 toneladas (Faostat, 2020). Tiene su centro de origen en el continente americano, con una amplia diversidad genética en Colombia, Ecuador, Norte del Perú, México y Centro América. Debido a su alto rendimiento, tanto de tubérculos como de follaje, bajo costo de producción y alto contenido nutricional, sobresale como un cultivo clave para la seguridad alimentaria, en diversas zonas del mundo (Castillo et al. 2014).

I. batatas es hexaploide $(2 \mathrm{n}=6 \mathrm{X}=90)$ y dependiendo del cultivar, las raíces tuberosas presentan variabilidad fenotípica, en tamaño, en forma (redondas, ronda elíptica, oval, obovado, largo oblongo, largo elíptico, largo irregular), en piel y en color del parénquima vascular de reserva (blanco, crema, amarillo, naranja, rojo o púrpura); también, se presenta variabilidad en las formas foliares: lobular, triangular, cordada, lanceolada y redonda (MartínezMoreno et al. 2018).

De manera tradicional, las hojas de batata cocinadas han sido empleadas como remedio para tumores en boca y garganta y también son utilizadas como astringente, tónico, laxante y fungicida; tienen un alto contenido en proteínas, vitaminas, minerales, fibra dietética y compuestos antioxidantes, como ácidos fenólicos, antocianinas, tocoferoles y betacarotenos (Grace et al. 2015; Nguyen et al. 2021). Según Flórez-Martínez et al. (2016), los tubérculos y las hojas pueden ser empleados en la alimentación de ovinos, caprinos, bovinos y peces; se puede administrar en fresco o generar alimentos balanceados. Las hojas expresan proteína cruda de 260-330 g. $\mathrm{kg}^{-1}$, de materia seca (MS), comparado con 100-140 g. $\mathrm{kg}^{-1}$ de MS, en los tallos; el contenido de proteína cruda (hojas y tallos) es de 16 a $29 \%$ sobre la materia seca, que es similar a las leguminosas forrajeras (Van et al. 2003).
El tubérculo es considerado un alimento funcional, utilizado para el tratamiento de enfermedades crónico-degenerativas, disminuyendo su incidencia y su prevalencia; tiene propiedades cardioprotectoras, anticancerígenas, antienvejecimiento, entre otras (Wang et al. 2016). Además, no solo son una buena fuente de energía, debido al contenido de carbohidratos, sino también para la producción de almidón y de alcohol, en medio fermentativo (Murillo-Martínez et al. 2021; Rizzolo et al. 2021).

El almidón de batata está conformado, en su mayor parte, por amilopectina (Lai et al. 2015), posee un patrón de tipo A (alto hinchamiento) y sus gránulos de son de tamaño mediano, con una forma oval redonda suave (Moorthy, 2002). Las aplicaciones industriales de almidón dependen de las propiedades de gelatinización, retrogradación, solubilidad, hinchamiento, absorción de agua, sinéresis y comportamiento reológico de pastas y geles (Murillo-Martínez et al. 2021). En cuanto a contenido nutricional, la batata es un alimento de alta energía, cuyos tubérculos tienen un contenido de carbohidratos totales de 25 a $30 \%$, de los cuales, el $98 \%$ son considerados altamente digeribles (Grüneberg et al. 2015).

El objetivo de esta investigación fue evaluar el rendimiento agronómico en valor relativo del forraje (VRF), rendimiento y calidad de almidón en tubérculos de batata.

\section{MATERIALES Y MÉTODOS}

Localización. El trabajo de campo, se realizó en el Centro Experimental Mario González Aranda, de la Universidad Nacional de Colombia, Sede Palmira, Valle del Cauca, situado a $03^{\circ} 30^{\prime 2} 26,8^{\prime \prime}$ $\mathrm{N}$ y 76²'47,6" O y altitud de 998 m s.n.m., temperatura promedio de $24{ }^{\circ} \mathrm{C}$, humedad relativa del $72 \%$ y precipitación pluvial anual de $1.000 \mathrm{~mm}$ (Valdés Restrepo et al. 2014). La valoración macromolecular, se hizo en el Laboratorio de Semillas y Laboratorio de Nutrición Animal y la prueba granulométrica, en el Laboratorio de Física de Suelos, de la misma Universidad, a $3^{\circ} 51^{\prime} 48^{\prime \prime} \mathrm{N}$ y $76^{\circ} 81^{\prime} 13^{\prime \prime} \mathrm{O}$, en el Valle del Cauca (Colombia) (Ordoñez Narváez et al. 2014).

Material genético. Se evaluaron 10 clones de batata (Tainug-66, 440-016, 440-045, 440-287, 440-078, 440-260, 440-255, 440-357, 440-621, 440-656), donados por CIAT, utilizando un diseño de bloques completos al azar, con 3 repeticiones y 6 plantas por repetición. Se utilizó un total de 180 plantas, a una distancia de siembra entre surcos de $2 \mathrm{~m}$ y dentro de surco, $1 \mathrm{~m}$ entre planta.

El modelo empleado fue el siguiente: $Y_{\mathrm{ij}}=\mu+\tau_{\mathrm{i}}+\beta_{\mathrm{j}}+\varepsilon_{\mathrm{ij}}$

Donde:

$Y_{\mathrm{ij}}=$ Variable de respuesta

$\mu=$ Media general

$\tau_{\mathrm{i}}=$ Efecto del i-ésimo tratamiento 
$\beta_{j}=$ Efecto del j-ésimo bloque

$\varepsilon_{i j}=$ Error experimental del tratamiento i en el bloque j

Caracteres evaluados en forraje. En forraje, se midió el rendimiento de forraje fresco disponible (t.ha $\left.{ }^{-1}\right)$ (RFF), rendimiento de forraje seco $\left(\right.$ t.h $\left.^{-1}\right)$ (RFS), materia seca del forraje de batata $(\%)$ (MSF), cenizas (\%), proteína (\%), extracto etéreo (\%), fibra detergente neutra FDN (\%), fibra detergente ácida FDA (\%) y el índice del valor relativo del forraje (VRF), el cual, se estima calidad, mediante el conocimiento de la digestibilidad de la materia seca (DMS), con base en la fibra detergente ácida (FDA), lo que facilita calcular la ingesta de la materia seca (IMS), con base en el contenido de (FDN). El índice, se calculó como el producto entre DMS y IMS dividido por 1,29 (Moore \& Undersander, 2001), así:

$$
\begin{aligned}
& \text { DMS }=88,9-(0,779 \times \% \text { FDA }) \\
& \text { IMS }=120 /(\% \text { FDN }) \\
& \text { VRF }=(\text { DMS } \times \text { IMS }) / 1,29
\end{aligned}
$$

Caracteres evaluados en tubérculo. Se registró el peso del tubérculo por cada $2 \mathrm{~m}^{2}(\mathrm{~kg})$, en términos de aforo de forraje fresco (AFFD), materia seca del tubérculo (\%) (MST), rendimiento del tubérculo $\left(\mathrm{t} . \mathrm{ha}^{-1}\right)(\mathrm{RT})$, cenizas (\%), extracto etéreo (\%), proteína $(\%)$, fibra detergente neutra FDN (\%) y fibra detergente ácida FDA (\%). Se determinó tres categorías en el tubérculo A, B, C (kg), siendo la categoría $\mathrm{A}$, los tubérculos que presentaron mayor peso; categoría $\mathrm{B}$, peso intermedio entre A y C y categoría C, aquellos de menor peso con formas variadas.

Para identificar los clones sobresalientes con respecto a los tubérculos, se utilizó el Índice de Selección Ponderado (ISP) (Ortiz-Grisales et al. 2013), donde participaron los caracteres: fibra detergente neutra (\%) (FDN), factor de ponderación $(0,5)$ y fibra detergente ácida (\%) (FDA), factor de ponderación $(0,5)$. El ISP para cada clon, se estimó a partir de la siguiente expresión:

$$
\operatorname{ISP}_{(k)}=\sum_{1}^{10} \operatorname{Pi} \frac{\bar{x}_{i(k)}-\bar{x}_{g(i)}}{S_{g(i)}}
$$

Dónde:

$\mathrm{ISP}_{(\mathrm{k})}=$ Índice de selección ponderado estandarizado para el k-ési-

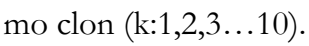

$P_{i}=$ Factor de ponderación asignado a la i-ésima variable (i: 1, 2, 3).

$\bar{X}_{i(k)}=$ Promedio de la i-ésima variable en el k-ésimo clon.

$\bar{X}_{g(k)}=$ Promedio general de la i-ésima variable en la colección.

$\mathrm{S}_{\mathrm{g}(i)}=$ Desviación estándar general de la i-ésima variable.
Establecimiento en campo y evaluación de clones. Se estableció un vivero, mediante la siembra del esqueje de batata, en vasos de polipropileno de $250 \mathrm{~mL}$, sobre una mezcla de compost, suelo y arena, en proporción 1:2:1, para un total de seis vasos por cada clon, los cuales, fueron trasplantados al lote experimental a los 43 días. El manejo agronómico fue el recomendado por el Programa de Hortalizas de la Universidad Nacional de Colombia, sede Palmira. Se tomó una muestra de $300 \mathrm{~g}$ de forraje por cada clon, para la determinación del análisis de fibras detergente neutra y análisis Weende, para la prueba de materia seca; las muestras fueron pesadas y depositadas en bolsas de papel, para la determinación de la materia seca (MS), mediante la técnica de secado en estufa, a $105^{\circ} \mathrm{C}$, por 24 h, según el protocolo de Ortiz-Grisales et al. (2013). La evaluación del forraje y cosecha de los frutos por planta, se realizó a los 80 días después de siembra.

Análisis químicos. El análisis proximal (Weende), se realizó en muestras liofilizadas y molidas, mediante descripción cuantitativa; las pruebas fueron: proteína cruda (PC), por el método Kjeldahl (AOAC, 1984); materia seca (MS), método 934.01 (AOAC, 1990); extracto etéreo (EE), método 920.39 (AOAC, 1990); cenizas, método 942.05 (AOAC, 1984; AOAC, 1990) y el análisis de fibra detergente neutra (FDN), se hizo por la tecnología Ankom (Van Soest et al. 1991).

Análisis de viscoamilógrama. El almidón de batata, se obtuvo por extracción manual, sobre el cual, se realizó la detección de temperatura de gelatinización y las curvas de empastamiento, que se determinaron utilizando un viscoamilógrafo, que establece el comportamiento reológico del almidón; las unidades de medición fueron centipoise (cP).

Para las condiciones de tiempo-temperatura, que garantizan la completa gelatinización de los almidones, se preparó una solución de almidón al $6 \%(\mathrm{p} / \mathrm{p})$, en base seca y agua destilada; el perfil de temperatura utilizado para el análisis de los almidones fue con una temperatura de inicio de $50{ }^{\circ} \mathrm{C}$, con un incremento de $7,5^{\circ} \mathrm{C} / \mathrm{min}$, hasta alcanzar los $92{ }^{\circ} \mathrm{C}$, el cual, se sostuvo por 5 minutos y un enfriamiento de $-7,5^{\circ} \mathrm{C} / \mathrm{min}$, hasta bajar la temperatura a $50{ }^{\circ} \mathrm{C}$ y se sostuvo por 3 minutos, $250 \mathrm{rpm}$ y $235 \mathrm{cmg}$.

Para identificar los clones sobresalientes respecto a la calidad del almidón, se utilizó el Índice de Selección Ponderado (ISP) (OrtizGrisales et al. 2013), donde participaron los caracteres: rendimiento del tubérculo (t.ha $\left.{ }^{-1}\right)(\mathrm{RT})$, factor de ponderación $(0,5)$ y materia seca del tubérculo (\%) (MST), factor de ponderación $(0,5)$.

Análisis estadístico. Se realizó el análisis de varianza (ANDEVA), a un nivel del $95 \%$ de confianza y para establecer diferencias significativas, se empleó el método de Duncan ( $\mathrm{p} \leq 0,05)$, para cada una de las variables en los 10 clones y para el tratamiento de la información, se utilizó el software SPSS (versión 21 Windows) y Microsoft Office, Excel 2010. 


\section{RESULTADOS Y DISCUSIÓN}

Forraje de batata. Los clones de batata presentan, en AFFD, un rendimiento que oscila entre 7,40 y $12,7 \mathrm{~kg} .\left(\mathrm{m}^{2}\right)^{-1}$, con un estimado de 49 t.ha $^{-1}$ y 10,63 \% de rendimiento de forraje fresco (RFF) y materia seca del fruto (MSF), respectivamente, donde se determinó que el clon 440-287 presenta el mejor RFF, con 63,67 tha ${ }^{-1}$ (Tabla 1). Estos rendimientos son superiores a $10,3 \%$ y 12,1 t.ha $^{-1}$, para MSF y RFF, determinado por Lam (2016).
Los 10 clones presentaron un contenido de materia seca promedio de 10,63\%, que se considera moderada, comparado con los reportados por Pico et al. (2011), quienes encontraron un 18,49\% de materia seca, con un alto contenido de minerales, representado en cenizas, con promedio de $12,87 \%$, proteína promedio de 14,19 $\%$, FDN de $50,23 \%$ y FDA de $31,97 \%$, lo que indica que, estos clones, se encuentran en el rango de materiales como la alfalfa fresca y la planta entera de maíz, con 21,7 y $10,5 \%$ de proteína, respectivamente. Du et al. (2016) reportan, para alfalfa fresca y la

Tabla 1. Rendimiento y calidad integral del forraje de batata.

\begin{tabular}{|c|c|c|c|c|c|c|c|c|c|c|c|c|}
\hline Introducción & $\begin{array}{c}\text { Aforo } \\
\text { Forraje } \\
\text { Fresco (kg) } \\
\text { (AFFD)* }\end{array}$ & $\begin{array}{c}\text { Rendimiento de } \\
\text { Forraje Fresco } \\
\left(\text { t.ha }^{-1}\right) \\
(\text { RFF })\end{array}$ & $\begin{array}{c}\text { Materia } \\
\text { Seca del } \\
\text { forraje (\%) } \\
\text { (MSF) }\end{array}$ & $\begin{array}{l}\text { Forraje seco } \\
\left(\text { t.ha }^{-1}\right)(\text { RFS })\end{array}$ & $\begin{array}{c}\text { Cenizas } \\
(\%)\end{array}$ & $\begin{array}{l}\text { PC } \\
(\%)\end{array}$ & $\begin{array}{l}\mathrm{EE} \\
(\%)\end{array}$ & $\begin{array}{c}\text { FDN } \\
(\%)\end{array}$ & $\begin{array}{c}\text { FDA } \\
(\%)\end{array}$ & $\begin{array}{c}\text { DMS } \\
(\%)\end{array}$ & IMS & VRF \\
\hline 440-045 & 11,34 & 56,68 & 9,50 & 5,04 & 13,07 & 12,21 & 3,78 & 48,40 & 30,40 & 65,22 & 2,48 & 125,34 \\
\hline $440-621$ & 9,63 & 48,17 & 12,30 & 5,90 & 12,55 & 13,42 & 3,24 & 54,17 & 33,99 & 62,42 & 2,22 & 107,19 \\
\hline $440-255$ & 10,23 & 51,17 & 12,05 & 6,20 & 11,94 & 14,02 & 4,03 & 48,49 & 30,46 & 65,17 & 2,47 & 125,03 \\
\hline $440-078$ & 9,85 & 49,25 & 9,97 & 4,92 & 11,33 & 12,84 & 5,78 & 46,30 & 29,10 & 66,23 & 2,59 & 133,07 \\
\hline $440-287$ & 12,73 & 63,67 & 10,95 & 6,90 & 13,80 & 16,58 & 2,49 & 50,81 & 31,90 & 64,05 & 2,36 & 117,26 \\
\hline $440-357$ & 11,57 & 57,83 & 9,08 & 5,36 & 15,68 & 15,33 & 3,41 & 48,98 & 30,76 & 64,93 & 2,45 & 123,32 \\
\hline $440-260$ & 8,55 & 42,75 & 11,09 & 3,14 & 12,39 & 13,01 & 1,96 & 50,57 & 31,75 & 64,16 & 2,37 & 118,03 \\
\hline $440-016$ & 9,14 & 45,72 & 11,09 & 5,02 & 11,69 & 14,04 & 1,82 & 53,01 & 33,27 & 62,98 & 2,26 & 110,52 \\
\hline $440-656$ & 7,50 & 37,50 & 9,27 & 3,48 & 12,72 & 15,43 & 3,81 & 54,82 & 34,39 & 62,11 & 2,19 & 105,39 \\
\hline Tainug-66 & 7,40 & 37,00 & 10,94 & 4,08 & 13,54 & 15,04 & 2,41 & 53,72 & 33,71 & 62,64 & 2,23 & 108,47 \\
\hline Promedios & 9,79 & 48,97 & 10,63 & 5,00 & 12,87 & 14,19 & 3,27 & 50,93 & 31,97 & 63,99 & 2,36 & 117,36 \\
\hline $\operatorname{DMS}_{(5 \%)}$ & 3.95 & 19,78 & 2,31 & 1,96 & 1,17 & 1,28 & 1,10 & 2,70 & 3,27 & 2.55 & 0,24 & 16,91 \\
\hline CV \% & 24.56 & 24,56 & 15,50 & 32,11 & 9,77 & 9,70 & 36,17 & 5,68 & 5,63 & 2.19 & 5,72 & 7,92 \\
\hline Promax-DMS** & 8.78 & 43,89 & 9,99 & 4,94 & 14,51 & 15,3 & 4,68 & 52,12 & 31,12 & 63,68 & 2,35 & 116,16 \\
\hline
\end{tabular}

* foro del forraje en 2 metros cuadrados. **Promax - DMS = Diferencia entre el promedio del clon de mejor desempeño y la diferencia mínima significativa. Determina el límite por encima del cual se ubican los promedios de los clones que no difieren del de más alto desempeño. PC: Proteína cruda; EE: Extracto etéreo; FDN: Fibra detergente neutra; FDA; Fibra detergente ácida; DMS: Digestibilidad de la materia seca; IMS: Ingesta de la materia seca; VRF: Valor relativo del forraje.

planta de maíz entera, un porcentaje de FDN de 35,7 y 72,9 \% y FDA de 23,1 y 40,8 \%; estos valores altos deprimen su consumo en animales de digestión enzimática. También es un follaje bajo en grasas; Wang et al. (2016) reportaron contenidos de grasa entre 0,4 y $3 \%$, similares al promedio de los 10 clones, con 3,27\%. De la tabla 1 , se deduce que el mejor clon para producción de forraje es el 440-287, dado que presenta buen desempeño en el aforo en fresco, rendimiento en fresco y seco, cenizas y proteína.

Con base en VRF, se determinó que el clon 440-078 expresó un valor de 133,07, seguido por los clones 440-045 y 440-255, que los ubica como los de mejor calidad integral, con un VRF de 125, si se compara con lo reportado por Putman et al. (2008), para alfalfa, que expresa un valor medio -VRF de 150, con un tope "Supreme" $>185$ unidades de -VRF. Por tanto, el forraje de batata puede ser ofrecido a rumiantes mayores (bovinos-vacas y búfalos) y menores (ovinos, caprinos), así como para ser ofertado a animales de digestión enzimática, previo deshidratado (Solis \& Ruiloba, 2017).

Tubérculo de batata. La materia seca varía, según el clon, el promedio de los 10 clones fue de 32,06\%, una MS que se encuentra acorde con los datos reportados por Martí (2018) y García-Méndez et al. (2016), quienes reportan una materia seca en el tubérculo de batata del 29,5 y $28,39 \%$, respectivamente.

Según el ISP de 1,32, el mejor clon de batata fue el 440-656, con alto rendimiento de $\left(\right.$ t.ha $\left.^{-1}\right)$ y alta materia seca (Tabla 2). El rendimiento para los clones oscila entre 21,19 y 35,45 t.ha $^{-1}$; aunque la variación es amplia, está acorde con los datos registrados por Šlosár et al. (2016), quienes reportan, para los cultivares comerciales Beauregard, Serbian y Zagrebian, rendimientos de 32,76, 39,30 y 32,01 th.ha ${ }^{-1}$, respectivamente.

El contenido de ceniza de los tubérculos de batata (Tabla 3) oscila entre 3,46 y $4,80 \%$, superiores a los datos reportados por García-Méndez et al. (2016), con 1,53\%, en promedio de cenizas y González et al. (2015), con 1,24 \%, en batata roja y $0,15 \%$, en batata amarilla.

El porcentaje promedio de proteína en los tubérculos fue de 5,25\% y grasa $2,02 \%$, datos superiores comparados con los reportados por Martí et al. (2011), valores de 1,63 y 0,60\%, respectivamente. 
Tabla 2. Rendimiento promedio e índice de selección ponderado en tubérculos de batata.

\begin{tabular}{|l|c|c|c|c|}
\hline Nombre Introducción & $\begin{array}{c}\text { Materia Seca del } \\
\text { Tubérculo (\%) (MST) }\end{array}$ & $\begin{array}{c}\text { Rendimiento de } \\
\text { Tubérculo (t.ha-1) } \\
\text { (RT) }\end{array}$ & ISP & Posición \\
\hline $440-656$ & 38,01 & 33,54 & 1,32 & 1 \\
\hline $440-621$ & 31,41 & 35,45 & 0,57 & 2 \\
\hline Tainug-66 & 32,39 & 33,49 & 0,51 & 3 \\
\hline $440-078$ & 35,04 & 27,08 & 0,26 & 4 \\
\hline $440-016$ & 31,62 & 28,84 & $-0,06$ & 5 \\
\hline $440-045$ & 33,5 & 25,66 & $-0,10$ & 6 \\
\hline $440-287$ & 26,44 & 33,29 & $-0,36$ & 7 \\
\hline $440-260$ & 30,33 & 27,61 & $-0,36$ & 8 \\
\hline $440-255$ & 34,59 & 21,19 & $-0,39$ & 9 \\
\hline $440-357$ & 27,26 & 21,75 & $-1,38$ & 10 \\
\hline Promedio & 32,06 & 28,79 & & \\
\hline DMS \% & 10,02 & 4,72 & & \\
\hline CV \% & 20,10 & 17,58 & & \\
\hline
\end{tabular}

Tabla 3. Rendimiento y contenido nutricional del tubérculo de batata.

\begin{tabular}{|c|c|c|c|c|c|c|c|c|c|c|c|}
\hline Introducción & $\begin{array}{c}\text { Nombre } \\
\text { Introducción }\end{array}$ & $\begin{array}{c}\text { Cenizas de } \\
\text { la Harina } \\
\text { de Batata } \\
(\%)\end{array}$ & $\begin{array}{c}\text { Proteína } \\
\text { Cruda de la } \\
\text { Harina de } \\
\text { Batata (\%) }\end{array}$ & $\begin{array}{c}\text { Extracto } \\
\text { Etéreo de } \\
\text { la Harina } \\
\text { de Batata } \\
(\%)\end{array}$ & $\begin{array}{c}\text { FDN } \\
(\%)\end{array}$ & $\begin{array}{c}\text { FDA } \\
\%\end{array}$ & ISP & Posición & $\begin{array}{c}\text { Peso Categoría } \\
\text { A (Kg) parcela } \\
\text { de } 1 \text { X } 5 \mathrm{~m}\end{array}$ & $\begin{array}{c}\text { Peso } \\
\text { Categoría B } \\
(\mathrm{Kg}) \text { parcela } \\
\text { de } 1 \text { X } 5 \mathrm{~m}\end{array}$ & \begin{tabular}{|c|} 
Peso \\
Categoría \\
C (Kg) \\
parcela de 1 \\
X 5 m \\
\end{tabular} \\
\hline 1 & $440-045$ & $3,82 \mathrm{c}$ & $5,97 \mathrm{abc}$ & $0,50 \mathrm{a}$ & $32,56 \mathrm{ab}$ & 23,32 & $-0,51$ & 8 & $21,30 \mathrm{bc}$ & $9,38 \mathrm{~b}$ & $5,25 \mathrm{~d}$ \\
\hline 2 & $440-621$ & $3,93 \mathrm{bc}$ & $4,51 \mathrm{de}$ & $2,86 a$ & $34,94 \mathrm{ab}$ & 25,29 & 0,14 & 4 & $38,28 \mathrm{a}$ & $16,43 \mathrm{a}$ & $16,43 \mathrm{a}$ \\
\hline 3 & $440-255$ & $3,93 \mathrm{bc}$ & $3,89 \mathrm{e}$ & $3,22 \mathrm{a}$ & $34,65 \mathrm{ab}$ & 25,07 & 0,07 & 5 & $23,07 \mathrm{bc}$ & $11,13 \mathrm{~b}$ & $10,60 \mathrm{bc}$ \\
\hline 4 & $440-078$ & $4,15 \mathrm{bc}$ & $4,79 \mathrm{de}$ & $1,70 \mathrm{a}$ & $34,26 \mathrm{ab}$ & 24,73 & $-0,04$ & 6 & $19,18 \mathrm{bc}$ & $10,05 \mathrm{~b}$ & $8,43 \mathrm{bcd}$ \\
\hline 5 & $440-287$ & $5,78 \mathrm{a}$ & $5,29 \mathrm{bcd}$ & $1,79 \mathrm{a}$ & $36,04 \mathrm{ab}$ & 26,21 & 0,45 & 3 & $28,88 \mathrm{ab}$ & $12,55 \mathrm{~b}$ & $12,28 \mathrm{ab}$ \\
\hline 6 & $440-357$ & $4,80 \mathrm{~b}$ & $6,19 \mathrm{ab}$ & $1,21 \mathrm{a}$ & $42,02 \mathrm{a}$ & 31,2 & 2,11 & 1 & $16,52 \mathrm{c}$ & $8,23 \mathrm{~b}$ & $6,90 \mathrm{~cd}$ \\
\hline 7 & $440-260$ & $4,28 \mathrm{bc}$ & 4,95 cde & $1,83 \mathrm{a}$ & $33,61 \mathrm{ab}$ & 24,28 & $-0,21$ & 7 & $18,35 \mathrm{bc}$ & $12,55 \mathrm{~b}$ & $7,13 \mathrm{~cd}$ \\
\hline 8 & $440-016$ & $3,46 \mathrm{c}$ & $4,07 \mathrm{e}$ & $2,03 \mathrm{a}$ & $30,35 \mathrm{~b}$ & 21,47 & $-1,13$ & 9 & $22,4 \mathrm{bc}$ & $11,83 \mathrm{~b}$ & $9,55 \mathrm{bcd}$ \\
\hline 9 & $440-656$ & $3,99 \mathrm{bc}$ & $6,69 \mathrm{a}$ & $3,05 \mathrm{a}$ & $36,76 \mathrm{ab}$ & 26,81 & 0,65 & 2 & $23,48 \mathrm{bc}$ & $10,58 \mathrm{~b}$ & $8,73 \mathrm{bcd}$ \\
\hline 10 & Tainug-66 & $4,07 \mathrm{bc}$ & $6,14 \mathrm{ab}$ & $1,97 \mathrm{a}$ & $28,89 \mathrm{~b}$ & 20,25 & $-1,53$ & 10 & $22,93 \mathrm{bc}$ & $9,65 \mathrm{~b}$ & $7,90 \mathrm{bcd}$ \\
\hline Promedio & & 4,22 & 5,25 & 2,02 & 34,41 & 24.86 & & & 23,44 & 11,24 & 9,32 \\
\hline DMS \% & & 0,90 & 1,09 & 2.61 & 9,16 & 5,47 & & & 11,17 & 5,02 & 4,97 \\
\hline $\mathrm{CV} \%$ & & 18,30 & 20,19 & 69,41 & 15,38 & 12,10 & & & 57,05 & 41,12 & 40,56 \\
\hline
\end{tabular}

FDN: Fibra detergente Neutra; FDA: fibra detergente ácida; ISP: Índice de selección ponderado.

Promedios con letras distintas indican diferencia significativa (Duncan) $(p \leq 0,05)$.

Estos autores afirman que la proteína de batata es valiosa, debido a su aporte de aminoácidos esenciales, destacándose la lisina, esto hace que la harina pueda ser utilizada como complemento de cereales deficientes en este aminoácido. A su vez, García-Méndez et al. (2016) identificaron en batata, variedad topera, niveles de proteína de $4,13 \%$, considerándolos altos y con potencialidad. Respecto a la FDN y FDA, se identificó en promedio 34,41 y 24,86 $\%$; estos valores, se consideran relevantes, con posibilidad de ser incorporados en una dieta animal, debido a su moderada FDN y FDA.
Se aclara que, a menor contenido de fibras (FDN y FDN) mayor calidad, valores bajos, muy bajos o negativos que se presenten en el índice de selección ponderado (ISP), hace referencia al clon sobresaliente, por tanto, el mejor material será el que exprese el valor más negativo. Según el índice de selección ponderado (ISP), el clon que presenta mejor calidad en el tubérculo es el Tainung-66, con ISP de -1,53 y bajo contenido de FDN y FDA. A su vez, el clon con más bajo desempeño fue 440-357, con un ISP de 2,11. 
Se identificaron tres categorías de calidad A, B y C, según su peso (Tabla 3). La categoría A, correspondiente al mayor peso de tubérculo, puede ser utilizada para procesos agroindustriales en la elaboración de harinas y derivos y las categorías B y C, para consumo en fresco. García-Méndez et al. (2016) afirman que la tendencia de los mercados va dirigida al consumo de raíces y de tubérculos de pesos livianos a medios y representan un menor aporte calórico y mayor digestibilidad.
Viscoamilograma de almidón de Batata. En la tabla 4, figura 1 , se presentan las propiedades funcionales y las curvas de empastamiento de almidón, en 10 clones de batata.

Temperatura de gelatinización. Se encontraron diferencias significativas en la temperatura inicial de empastamiento para los 10 clones de batata ( $\mathrm{p} \leq 0,05)$; los clones 440-621, 440-078 y 440-656 presentaron las más altas temperaturas de gelatinización (Tabla 4),

Tabla 4. Viscosidad del almidón de batata durante ciclos de calentamiento-enfriamiento ( \pm desviación estándar).

\begin{tabular}{|l|c|c|c|c|c|c|}
\hline Referencia & $\mathbf{A}$ & $\mathbf{B}$ & $\mathbf{C}$ & $\mathbf{D}$ & $\mathbf{E}$ & $\mathbf{F}$ \\
\hline $\mathbf{4 4 0 - 0 4 5}$ & $73,7 \pm 0,6 \mathrm{e}$ & $801 \pm 18,5 \mathrm{ab}$ & $540 \pm 33,9 \mathrm{~d}$ & $261 \pm 47,5 \mathrm{a}$ & $735 \pm 53,9 \mathrm{~d}$ & $194 \pm 27,5 \mathrm{~b}$ \\
\hline $\mathbf{4 4 0 - 6 2 1}$ & $75,4 \pm 0,5 \mathrm{a}$ & $822 \pm 36,2 \mathrm{a}$ & $595 \pm 17,9 \mathrm{bc}$ & $227 \pm 45,5 \mathrm{abc}$ & $781 \pm 12,2 \mathrm{~d}$ & $187 \pm 15,3 \mathrm{~b}$ \\
\hline $\mathbf{4 4 0 - 2 5 5}$ & $73,6 \pm 0,3 \mathrm{e}$ & $837 \pm 34,8 \mathrm{a}$ & $573 \pm 38,5 \mathrm{~d}$ & $265 \pm 58,1 \mathrm{a}$ & $755 \pm 35,6 \mathrm{~d}$ & $183 \pm 8,0 \mathrm{~b}$ \\
\hline $\mathbf{4 4 0 - 0 7 8}$ & $75,3 \pm 0,3 \mathrm{a}$ & $734 \pm 63,4 \mathrm{~d}$ & $605 \pm 10,4 \mathrm{abc}$ & $163 \pm 1,2$ & $797 \pm 30,5 \mathrm{abc}$ & $193 \pm 22,2 \mathrm{~b}$ \\
\hline $\mathbf{4 4 0 - 2 8 7}$ & $74,4 \pm 0,5 \mathrm{~b}$ & $777 \pm 22,8 \mathrm{~d}$ & $671 \pm 15,1 \mathrm{a}$ & $105 \pm 36,9 \mathrm{~d}$ & $869 \pm 16,7 \mathrm{a}$ & $197 \pm 9,8 \mathrm{~b}$ \\
\hline $\mathbf{4 4 0 - 3 5 7}$ & $74,3 \pm 0,2 \mathrm{bc}$ & $799 \pm 56,0 \mathrm{ab}$ & $639 \pm 55,9 \mathrm{ab}$ & $193 \pm 58,9$ & $834 \pm 62,1 \mathrm{abc}$ & $195 \pm 25,3 \mathrm{~b}$ \\
\hline $\mathbf{4 4 0 - 2 6 0}$ & $74,0 \pm 0,8 \mathrm{~cd}$ & $831 \pm 51,5 \mathrm{a}$ & $583 \pm 29,7 \mathrm{~d}$ & $248 \pm 79,7 \mathrm{ab}$ & $772 \pm 9,8 \mathrm{~d}$ & $190 \pm 23,0 \mathrm{~b}$ \\
\hline $\mathbf{4 4 0 - 0 1 6}$ & $73,3 \pm 0,1 \mathrm{e}$ & $795 \pm 9,2 \mathrm{abc}$ & $617 \pm 4,6 \mathrm{abc}$ & $178 \pm 9,0 \mathrm{~d}$ & $838 \pm 17,6 \mathrm{ab}$ & $221 \pm 14,7 \mathrm{a}$ \\
\hline $\mathbf{4 4 0 - 6 5 6}$ & $75,3 \pm 0,3 \mathrm{a}$ & $723 \pm 4,0 \mathrm{~d}$ & $609 \pm 12,2 \mathrm{abc}$ & $114 \pm 8,5 \mathrm{~d}$ & $806 \pm 19,8 \mathrm{abc}$ & $197 \pm 15,0 \mathrm{~b}$ \\
\hline Tainug-66 & $74,5 \pm 0,2 \mathrm{~b}$ & $730 \pm 12,3 \mathrm{~d}$ & $569 \pm 20,3 \mathrm{~d}$ & $162 \pm 22,7 \mathrm{~d}$ & $738 \pm 13,8 \mathrm{~d}$ & $169 \pm 24,5 \mathrm{~b}$ \\
\hline \multicolumn{1}{|c|}{ DMS } & 0,66 & & 50,30 & 79,93 & 56,90 & 34,051 \\
\hline
\end{tabular}

A: Temperatura de empastamiento $\left({ }^{\circ} \mathrm{C}\right)$; B: Viscosidad máxima $(\mathrm{cP})$; C: viscosidad de la pasta caliente VPC (cP); D: Inestabilidad del gel Vmax-VPC (cP); E: viscosidad de la pasta fría VPF (cP); F: Reorganización VPF-VPC.

Promedios con letras distintas indican diferencia significativa (Duncan) ( $\mathrm{p} \leq 0,05)$.

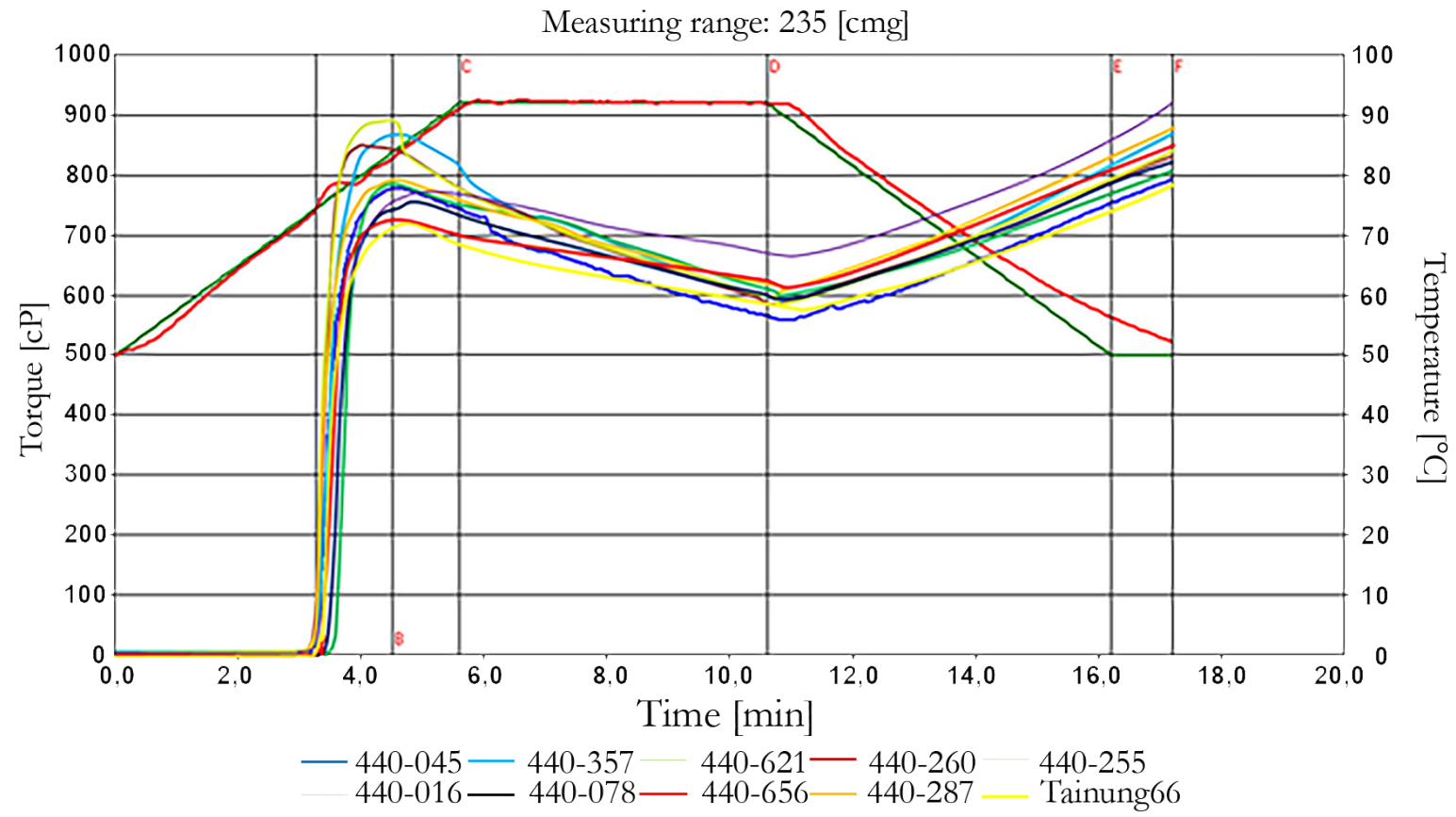

Figura 1. Viscoamilograma de almidones nativos de batata. 
lo que indica, que requieren mayor tiempo de cocción para iniciar el proceso de absorción de agua, hinchamiento y gelatinización. Estas temperaturas son coherentes con lo reportado por JiménezVillalba et al. (2019), en cuatro clones de batata, con temperaturas de gelatinización, que oscilan entre los 74 y $80^{\circ} \mathrm{C}$. El calor rompe los enlaces entre las micelas cristalinas del almidón, lo que hace que la amilosa se solubilice y se incremente la viscosidad; por tanto, entre mayor sea el tiempo y más alta la temperatura, el almidón demorará en alcanzar su viscosidad máxima, repercutiendo en la facilidad de cocción. Beleia et al. (2006) reportan que, a mayor contenido de amilosa mayor temperatura para iniciar el proceso de pastificación. Según Hoyos-Leyva et al. (2012), las bajas temperaturas de gelatinización, se explican porque el gránulo de almidón absorbe agua de manera rápida, debido al debilitamiento entre las fuerzas de atracción entre las moléculas (amilosa/amilopectina).

La viscosidad máxima (Vm). Los almidones evaluados presentaron una viscosidad máxima, que oscila entre 723 y $837 \mathrm{cP}$, arrojando diferencias significativas ( $\mathrm{p} \leq 0,05)$, entre ellos, estos resultados fueron consistentes con los reportados por Zhou et al. (2017), con valores de $745 \mathrm{cP}$ de viscosidad, máxima en almidones de batata. Los valores de Vm más bajos (Figura 1), se presentaron en los clones 440-656 y Tainug-66, con 723 y 730cP, respectivamente, los cuales, podrían ser empleados en la industria alimentaria en la elaboración de gomas y dulces, mientras que los clones 440-621, 440-255 y 440-260 tuvieron los más alto pico de viscosidad, posiblemente, a que los gránulos de almidón de estos clones tienen mayor absorción de agua al ser sometidos a calentamiento; según Beleia et al. (2006), a medida que los gránulos de almidón absorben agua y se hinchan, modifican su tamaño y volumen, va aumentando la viscosidad hasta alcanzar su pico máximo de viscosidad y los gránulos se rompen. Jiménez-Villalba et al. (2019) afirman que, entre más amilosa contengan los almidones, más dramático será su aumento en viscosidad y que la Vm de la pasta, durante el calentamiento, podría ser un buen espesante en la industria de confitería.

Viscosidad de la pasta caliente (VPC). La VPC, se debe a los gránulos hinchados, moléculas de almidón coloidal y dispersiones presentes en la pasta caliente (Zhou et al. 2016). Se encontraron diferencias significativas $(p<0,05)$ entre los clones; sin embargo, todos los clones tienen una marcada tendencia (Figura 1), al comparar la Vm con la VPC y la VPF.

La inestabilidad del gel (Vmax-VPC). Se evidenciaron diferencias significativas ( $\mathrm{p} \leq 0,05$ ) entre los clones (Tabla 4); 440255 y 440-045 presentaron los valores más altos de inestabilidad del gel 265 y $261 \mathrm{cP}$, respectivamente, lo que indica que no son pastas estables; según Paternina et al. (2016), la inestabilidad del gel es un indicativo de qué tan resistentes son los geles, al cizallamiento mecánico y térmico, en procesos agroindustriales. Los valores de inestabilidad del gel más bajos corresponden a los clones 440-287 y 440-656, con 105 y 114 cP, respectivamente.

La viscosidad de la pasta fría (VPF). Se encontraron diferencias significativas entre los clones; sin embargo, en los clones 440-078,
440-287, 440-357, 440-016 y 440-656, cuando la pasta se enfrió, la viscosidad aumentó, lo que indica que no son estables a los procesos de enfriamiento; este mismo fenómeno, se presentó en tubérculos de makal, yuca y sagú (Hernández-Medina et al. 2008).

Reorganización (VPF-Vmax). Variable asociada con la retrogradación, no se presentaron diferencias significativas ( $\mathrm{p} \leq 0,05)$, salvo en el clon 440-016, con $221 \mathrm{cP}$, indicando que este clon tiene tendencia a la retrogradación. Jiménez-Villalba et al. (2019) reportaron resultados superiores, entre 698 y $1239 \mathrm{cP}$, los cuales, variaron según la localidad y recomiendan realizar técnicas de modificación a la estructura del almidón nativo, debido a los altos resultados. La reorganización o Setback es importante, porque cuando el almidón se enfría y después se refrigera, adquiere una estructura rígida, la cual, se retrae y luego elimina agua. Por su parte, el clon Tainug-66 presentó una viscosidad máxima de $730 \mathrm{cP}$ y una viscosidad de la pasta fría de $738 \mathrm{cP}$, lo que lo hace el clon con la pasta más estable e interesante para la elaboración de productos que requieran un enfriamiento, congelación y refrigeración, como los flanes.

Conflicto de intereses: El manuscrito fue preparado y revisado con la participación de todos los autores, quienes declaramos que no existe ningún conflicto de intereses que ponga en riesgo la validez de los resultados presentados.

\section{REFERENCIAS}

1. ASSOCIATION OF OFFICIAL AGRICULTURAL CHEMISTRY, AOAC. 1984. Official Methods of Analysis. 10th. Ed. Association of Official Agricultural Chemistry. Washington.

2. ASSOCIATION OF OFFICIAL AGRICULTURAL CHEMISTRY, AOAC. 1990. Official Methods of analysis of the Association of Official analytical chemists. 15th Ed. Arlington (Virginia, USA). p.30-64.

3. BELEIA, A.; BUTARELO, S.; SILVA, R. 2006. Modeling of starch gelatinization during cooking of cassava (Manihot esculenta Crantz). Food Sci. Technol. 39:400-405.

https://doi.org/10.1016/j.lwt.2005.02.021

4. CASTILLO, M.R.; BRENES, A.A.; ESKER, P.; GÓMEZ, A.L. 2014. Evaluación agronómica de trece genotipos de camote (Ipomoea batatas L.). Agronomía Costarricense. 38(2):67-81.

5. DU, S.; XU, M.; YAO, J. 2016. Relationship between fibre degradation kinetics and chemical composition of forages and by-products in ruminants. J. Applied Animal Research. 44(1):189-193. https://doi.org/10.1080/09712119.2015.1031767

6. FAOSTAT. 2020. Food and agriculture Organization of the United Nations. Base de datos, cultivos (producción). Disponible desde internet en: http://www.fao.org/faostat/es/\#data/QC (con acceso el 
$26 / 07 / 2020)$

7. FLÓREZ-MARTÍNEZ, D.H.; URIBE-GALVIS, C.P.; CONTRERAS-PEDROZA， C.A. 2016. Perspectivas tecnológicas y comerciales para el cultivo de la batata en Colombia. Corporación Colombiana de Investigación Agropecuaria- Corpoica (Mosquera, Colombia). 110p.

8. GARCÍA-MÉNDEZ, A.D.; PÉREZ-DARNIZ, M.Y.; GARCÍAMENDEZ, A.A.; MADRIZ-IZTURIZ, P.M. 2016. Postharvest characterization and chemical composition of sweet potato (Ipomoea batatas (L.) Lamb.) Topera variety. Agronomía Mesoamericana. 27(2):287-300.

https://doi.org/10.15517/am.v27i2.21426

9. GONZÁLEZ, A.; ALVIS, A.; ARRÁZOLA, G. 2015. Efecto del recubrimiento comestible en las propiedades de trozos de batata (Ipomoea batatas Lam) fritos por inmersión: Parte 1: Textura. Información tecnológica. 26(1):95-102. https://dx.doi.org/10.4067/S0718-07642015000100011

10. GRACE, M.H.; TRUONG, A.N.; TRUONG, V.; RASKIN, I.; LILA, M.A. 2015. Novel value - added uses for sweet potato juice and flour in polyphenol - and protein - enriched functional food ingredients. Food Sci Nutr. 3(5):415-424. https://dx.doi.org/10.1002/fsn3.234

11. GRÜNEBERG, W.J.; MA, D.; MWANGA., R.O.M.; CAREY, E.E.; HUAMANI, K.; DIAZ, F.; EYZAGUIRRE, R.; GUAF, E.; JUSUF, M.; KARUNIAWAN, A.; TJINTOKOHADI, K.; SONG, Y.S.; ANIL, S.R.; HOSSAIN, M.; RAHAMAN, E.; ATTALURI, S.I.; SOMÉ, K.; AFUAPE, S.O.; ADOFO, K.; LUKONGE, E.; KARANJA, L.; NDIRIGWE, J.; SSEMAKULA, G.; AGILI, S.; RANDRIANAIVOARIVONY, J.M.; CHIONA, M.; CHIPUNGU, F.; LAURIE, S.M.; RICARDO, J.; ANDRADE, M.; RAUSCH, F.F.; MELLO, A.S.; KHAN, M.A.; LABONTE, D.R.; YENCHO, G.C. 2015. Advances in sweetpotato breeding from 1992 to 2012. In: Campos, H.; Caligari, P.D.S. (eds). Genetic improvement of tropical crops. Springer. Cham Switzerland. p.3-68.

12. HERNÁNDEZ-MEDINA, M.; TORRUCO-UCO, J.G.; CHEL-GUERRERO, L.; BETANCUR-ANCONA, D. 2008. Caracterización fisicoquímica de almidones de tubérculos cultivados en Yucatán, México. Food Science and Technology. 28(3):718-726.

https://doi.org/10.1590/S0101-20612008000300031

13. HOYOS-LEYVA, J.D.; JARAMILLO-JIMÉNEZ, P.A.; GIRALDO-TORO, A.; DUFOUR, D.; SÁNCHEZ, T.; LUCAS AGUIRRE, J.C. 2012. Caracterización física, morfológica y evaluación de las curvas de empastamiento de musáceas (Musa spp.). Acta Agronómica. 61(3):214-229.

14. JIMÉNEZ-VILLALBA， K.; ARRIETA-BANQUET， L.;
SALCEDO-MENDOZA, J.; CONTRERAS-LOZANO, K. 2019. Caracterización de harinas y almidones de batatas (Ipomoea batatas Lam.) de la costa caribe colombiana. Rev. U.D.C.A Act \& Div. Cient. 22(1):e1185.

https://doi.org/10.31910/rudca.v22.n1.2019.1185

15. KOUSSAO, S.; GRACEN, V.; ASANTE, I.; DANQUAH, E.Y.; OUEDRAOGO, J.T.; BAPTISTE, T.J.; JEROME, B.; VIANNEY, T.M. 2014. Diversity analysis of sweet potato (Ipomoea batatas [L.] Lam) germplasm from Burkina Faso using morphological and simple sequence repeats markers. African Journal of Biotechnology. 13(6):729-742. https://doi.org/10.5897/AJB2013.13234

16. LAI, Y.C.; WANG, S.Y.; GAO, H.Y.; NGUYEN, K.M.; NGUYEN, C.H.; SHIH, M.C.; LIN, K.H. 2015. Physicochemical properties of starches and expression and activity of starch biosynthesis-related genes in sweet potatoes. Food Chemistry. 199:556-564. https://doi.org/10.1016/j.foodchem.2015.12.053

17. LAM, V. 2016. Agricultural potential of sweet potato (Ipomoea batatas, L. (Lam)) for forage production. Livestock Research for Rural Development. 28:101.

18. MARTÍ, H.R. 2018. Producción de batata. 1ra. Edición, Ediciones INTA.

19. MARTÍ, H.R.; D’CHUDIL, H.; CORBINO, G. 2011. La batata: el redescubrimiento de un cultivo. Ciencia hoy. 21(121):17-23.

20. MARTÍNEZ-MORENO, D.; REYES-MATAMOROS, J.R.; BASURTO-PEÑA, F.B.; ANDRÉS-HERNÁNDEZ, R.A. 2018. Asignación de recursos en camote (Ipomoea batatas). Rev. Iberoam. Cienc. 5(4):8-18.

21. MOORE, J.E.; UNDERSANDER, D.J. 2001. Relative Forage Quality: An Alternative to Relative Feed Value and Quality Index. Proceedings 13th Annual Florida Ruminant Nutrition Symposium. p.16-32.

22. MOORTHY, S.N. 2002. Physicochemical and functional properties of tropical tuber starches: A review. StarchStarke. 54(12):559-592. https://doi.org/10.1002/1521379X(200212)54:12<559::A ID-STAR2222559>3.0.CO;2-F

23. MURILLO-MARTÍNEZ, M.; ALVIS-BERMÚDEZ, A.; ARRAZOLA-PATERNINA G. 2021. Physicochemical and functional properties of almidon obtained from two varieties of sweet potatoes (Ipomoea batatas). Rev. Bio. Agro. 19(1):117-127. https://doi.org/10.18684/bsaa(19)117-127

24. NGUYEN, H.C.; CHEN, C.-C.; LIN, K.-H.; CHAO, P.-Y.; 
LIN, H.-H.; HUANG, M.Y. 2021. Bioactive Compounds, Antioxidants, and Health Benefits of Sweet Potato Leaves. Molecules. 26(7):1820.

https://doi.org/10.3390/molecules26071820

25. ORDOÑEZ NARVÁEZ, G.A.; ORTIZ GRISALES, S.; VALDÉS RESTREPO, M.P.; VALLEJO CABRERA, F.A. 2014. Selección de introducciones de Cucurbita por contenido de aceite en semillas. Acta Agronómica. 63(2):175-180. https://doi.org/10.15446/acag.v63n2.40026

26. ORTIZ-GRISALES, S.; VALLEJO-CABRERA, F.A.; BAENA-GARCIA， G.D.; ESTRADA-SALAZAR, E.I.; VALDÉS-RESTREPO, M.P. 2013. Zapallo para consumo en fresco y fines agroindustriales: Investigación y desarrollo. Universidad Nacional de Colombia, Sede Palmira. Santiago de Cali, Feriva. Colombia. ISBN: 978-958-761-509-8.

27. PATERNINA C., A.; SALCEDO, M.J;; ROMERO, B.P. 2016. Efecto de la harina de ñame (Dioscorea rotundata P.) sobre las propiedades texturales de salchichas. Agronomía Colombiana. Suplemento 1:S379-S381.

https://doi.org/10.15446/agron.colomb.sup.2016n1.58021

28. PICO F., S.M.; GUTIÉRREZ, D.; ARAGÓN, G.I.; ESCOBAR, S.A.; ORTIZ, D.A.; SÁNCHEZ, T.; IMBACHÍ N., P.; PACHÓN, H. 2011. Evaluación de la composición nutricional, antinutricional y biodisponibilidad in vitro de diferentes extractos foliares. Rev. chilena de nutrición. 38(2):168-176.

https://dx.doi.org/10.4067/S0717-75182011000200007

29. PUTMAN, D.H.; ROBINSON, P.; PETERS, DE E. 2008. Forage Quality Testing. Chapter 16. In: Irrigated alfalfa management for Mediterranean and desert zones._Disponible desde internet en:

https://alfalfa.ucdavis.edu/IrrigatedAlfalfa/pdfs/UCAlfalf a8302ForageQuality_free.pdf (con acceso el 26/07/2020)

30. RIZZOLO, J.A.; WOICIECHOWSKI, A.L.; MAGALHÃES JÚNIOR, A.I.; ZEVALLOS TORRES, L.A.; SOCCOL, C.R. 2021. The potential of sweet potato biorefinery and development of alternative uses. SN Appl. Sci. 3:347. https://doi.org/10.1007/s42452-021-04369-y

31. ŠLOSÁR, M.; MEZEYOVÁ, I.; HEGEDÜSOVÁ, A.; GOLIAN, M. 2016. Sweet potato (Ipomoea batatas L.) growing in conditions of southern slovak republic. Potravinarstvo. 10(1):384-392.

http://dx.doi.org/10.5219/626

32. SOLIS, C.; RUILOBA, M.H. 2017. Evaluación de diferentes niveles de ensilaje integral de camote (Ipomoea batatas) como fuente energética para bovinos en crecimiento. Cuban J. Agric. Sci. 51(1):35-46.

33. VALDÉS RESTREPO, M.P.; ORTIZ GRISALES, S.; VALLEJO CABRERA, F.A.; BAENA GARCÍA, D. 2014. Variabilidad en frutos y semillas de Cucurbita moschata Duch. y Cucurbita argyrosperma subsp. sororia L.H. Bailey Merrick \& D.M. Bates. Acta Agronómica. 63(3):282-293.

http://dx.doi.org/10.15446/acag.v63n3.41052

34. VAN, A.L.; LINDBERG, B.E.F.; LINDBERG, J.E. 2003. Effect of harvesting interval and defoliation on yield and chemical composition of leaves, stems and tubers of sweet potato (Ipomoea batatas L (Lam.)) plant parts. Field Crops Res. 82(1):49-58. https://doi.org/10.1016/S0378-4290(03)00018-2

35. VAN SOEST, P.J.; ROBERTSON, J.B.; LEWIS B.A. 1991. Methods for dietary fiber, neutral detergent fiber, and nonstarch polysaccharides in relation to animal nutrition. J. Dairy Sci. 74(10):3583-3597. https://doi.org/10.3168/jds.S0022-0302(91)78551-2

36. WANG, S.; NIE, S.; ZHU, F. 2016. Chemical constituents and health effects of sweet potato. Food Res Int. 89(1):90-116. https://doi.org/10.1016/j.foodres.2016.08.032

37. ZHOU, D.N.; ZHANG, B.; CHEN, B.; CHEN, H.Q. 2017. Effects of oligosaccharides on pasting, thermal and rheological properties of sweet potato starch. Food Chemistry. 230:516-523. https://doi.org/10.1016/j.foodchem.2017.03.088

38. ZHOU, F.; LIU, Q.; ZHANG, H.; CHEN, Q.; KONG, B. 2016. Potato starch oxidation induced by sodium hypochlorite and its effect on functional properties and digestibility. Int. J. Biological macromolecules. 84:410-417. https://doi.org/10.1016/j.ijbiomac.2015.12.050 